\title{
PRDX6 alleviates lipopolysaccharide-induced inflammation in human gingival fibroblasts by regulation of NRF2
}

Wen-ying Yang ${ }^{1}$; Xiang Meng 1; Yue-rong Wang 1; Qing-qing Wang 1;2; Xin He 1;2; Xiao-yu Sun 1;2; Nan Cheng 1;2; Lei

1 College \& Hospital of Stomatology, Anhui Medical University, Key Lab. of Oral Diseases Research of Anhui Province, Hefei, 230032, China

2 Periodontal Department, Anhui Stomatology Hospital affiliated to Anhui Medical University, Hefei, 230032, China

* Correspondence: zhanglei6551@126.com

\begin{abstract}
Periodontitis is a progressive and inflammatory oral disease and results in the damage of the supporting tissues of teeth. Peroxiredoxin6 (PRDX6) is an antioxidant enzyme and has been identified as a regulator in redox balance. This study aimed to investigate whether PRDX6 could protect human gingival fibroblasts (HGFs) from lipopolysaccharide (LPS) induced inflammation and its mechanisms. Here, both inflamed and non-inflamed human gingival tissues were collected to assess the expression of PRDX6 and NRF2 by Immunohistochemistry and Western blotting. Furthermore, HGFs were stimulated with LPS, MJ33 (PRDX6 phospholipase A2 inhibitor), or ML385 (NRF2 inhibitor). The expression levels of inflammatory cytokines were measured by RT-qPCR and ELISA, and reactive oxygen species (ROS) was detected using DCFH-DA. PRDX6 was downregulated in inflamed gingival tissues. In HGFs, LPS induced inflammatory cytokines and ROS was upregulated in PRDX6 knockdown cells. Furthermore, co-treatment with MJ33 alleviated LPS-induced inflammatory cytokines and ROS, while inhibiting NRF2 upregulated those in HGFs. Therefore, this study provided a new mechanistic insight that PRDX6, regulated by the NRF2 signaling, alleviates LPS- induced periodontitis in human gingival fibroblasts.
\end{abstract}

Keywords: PRDX6; inflammation; NRF2; HGFs.

\section{Introduction}

Periodontitis is a chronic inflammatory disease of the periodontium and results in tooth loss [1, 2]. It affects $35 \%-50 \%$ of the adult population worldwide [3]. It is mainly caused by pathogenic bacteria-induced inflammatory responses and host immune responses [4-6]. Other risk factors of periodontitis include smoking, oxidative stress, systemic diseases, etc. [7]. Therefore, exploring the underlying mechanisms of periodontitis is of great significance. Reactive oxygen species (ROS) are critical in cell physiological processes, such as signal transduction, antimicrobial defense, and cellular transcription [8]. However, the overabundance of ROS production induced by lipopolysaccharide (LPS), a component of periodontal pathogenic bacteria, will cause tissue damage in periodontitis [9-11]. Besides, many reports demonstrated that some antioxidants could ameliorate LPSinduced periodontitis by minimizing oxidative stress [12-15].

Peroxiredoxins (PRDXs) are antioxidant enzymes that control intracellular peroxides level and mediate signal transduction. PRDX6 is an intriguing enzyme involved in many diseases, such as carcinogenesis, inflammation, ocular damage, etc. [16-20]. Unlike other peroxiredoxins, PRDX6 contains single cysteine and mainly acts PL hydroperoxide GPx (PHGPx) activity. Besides, it has two additional catalytic sites with phospholipase A2 activity (PLA2) activity and LPC acyl transferase (LPCAT) activity [21, 22]. Furthermore, 
PRDX6 is regulated by the antioxidant response element (ARE) downstream of the NRF2 (nuclear factor-erythropoietin 2-related factor 2, NRF2) transcription factor [23]. The microarray results showed that PRDX6 was upregulated in periodontitis [24]. However, the detailed function of PRDX6 and NRF2 in LPS-induced periodontitis has not been explored intensively.

This study aimed to explore the role of PRDX6 in modulating the inflammatory response and oxidative stress in periodontitis and its underlying mechanisms. Therefore, our study provided a new sight that PRDX6 plays a protective role in periodontitis through limiting oxidative stress.

\section{Materials and Methods}

\subsection{Reagents and antibodies}

E. coli LPS (L8880) was purchased from Solarbio (China) and dissolved in phosphatebuffered solution (PBS). MJ33 (C5326) and ML385 (B8300) were purchased from APEXBIO (USA) and dissolved in H2O and DMSO. PRDX6 monoclonal antibody (135851-AP, 1:1000) was purchased from Proteintech (China). NRF2 monoclonal antibody (12721S, 1:1000) was purchased from CST (USA). GAPDH monoclonal antibody (TA-08, 1:1000) was purchased from ZSGB-BIO (China).

\subsection{Participant recrument}

Gingival biopsies were obtained from healthy volunteers and chronic periodontitis patients at the Stomatology Hospital. The inclusion criteria for all volunteers in this study: (1) age $\geq 21$ years and the presence of 20 teeth; (2) volunteers without systemic diseases such as diabetes, rheumatoid arthritis, or coronary heart disease; (3) women without breastfeeding, menstruating, or pregnant; (4) no antibiotics, aspirin, or anticoagulants were used for recent three months; (5) without periodontal treatment within six months; (6) no habits of alcohol and tobacco. For patients with chronic periodontitis, additional criteria include (1) multiple sites with probing depth (PD) $\geq 5 \mathrm{~mm}$, (2) attachment loss (AL) $\geq 3 \mathrm{~mm}$, (3) bone loss visible on the radiograph. For healthy individuals, additional criteria include (1) without gingival inflammation, (2) without attachment loss, (3) probing depth $\leq 3 \mathrm{~mm}$, (4) no bleeding on probing. This study was conducted according to the guidelines approved by the Health Human Research Ethics Committee. All participants signed the written informed consent forms. The characteristics of control volunteers and chronic periodontitis patients are listed in Table 1.

Table 1. Characteristics of the study group.

\begin{tabular}{lll}
\hline Parameters & Controls & Patients \\
\hline Case, $\mathrm{n}$ & 16 & 16 \\
Age, $\mathrm{n}$ & $30.8 \pm 5.6$ & $32.3 \pm 6.2$ \\
Sex, $\mathrm{n}(\%)$ & & \\
Male & $7(44 \%)$ & $10(63 \%)$ \\
Female & $9(56 \%)$ & $6(37 \%)$ \\
Probing depth $(\mathrm{mm})$ & $2.07 \pm 0.29$ & $5.78 \pm 1.26$ \\
Attachment loss $(\mathrm{mm})$ & 0 & $4.81 \pm 1.38$ \\
Gingival index & 0 & $2.05 \pm 0.67$ \\
\hline
\end{tabular}

\subsection{Specimen collection}

Gingival biopsies were obtained from healthy volunteers $(n=16$; mean age $30.8 \pm 5.6$ years) and chronic periodontitis patients $(n=16$; mean age $32.3 \pm 6.2$ years). Healthy gingival tissues were obtained from patients undergoing crown-lengthening surgery with $\mathrm{PD} \leq 3 \mathrm{~mm}$ and without bleeding on probing. Gingival tissues of chronic periodontitis were collected from patients undergoing periodontal flap surgery with $P D \geq 5 \mathrm{~mm}$ and positive bleeding on probing. Each gingival tissue was equally divided into two sections. 
One section was used for hematoxylin \& eosin (H\&E) and Immunohistochemistry (IHC) analysis, and another section was frozen in liquid nitrogen for Western blotting.

\subsection{Immunohistochemistry (IHC)}

The gingival tissues were collected and fixed in $4 \%$ paraformaldehyde overnight. These specimens were embedded in paraffin, serially sectioned $(4 \mu \mathrm{m})$, and stained with H\&E. The IHC accessory kit (ZSGB- BIO, China) was used following the protocol provided. The sections were blocked with $0.03 \%$ hydrogen peroxide and $2 \%$ bovine serum albumin (BSA). Gingival specimens were incubated with antibodies against NRF2 and PRDX 6 at $4{ }^{\circ} \mathrm{C}$ for $12 \mathrm{~h}$, followed by incubation with secondary antibodies for $1 \mathrm{~h}$. Then the samples were counterstained with hematoxylin, dehydrated, and mounted. Morphological changes were observed by light microscopy (Leica, Germany).

\subsection{Cell culture}

HGFs were isolated from the non-inflamed gingiva of 5 donors who needed extraction of the healthy third molar. The cells were cultured in DMEM medium (Hyclone, USA) supplemented 10\% fetal bovine serum (Corning, USA) and 1\% penicillin/streptomycin solution (Beyotime, China) at $37{ }^{\circ} \mathrm{C}$ with $5 \% \mathrm{CO}_{2}$ in a cell incubator. Cells at 4-6 passages were used in the following experiment.

\subsection{Cell viability assay}

The CCK-8 (MCE, USA) method was used to access cell viability. HGFs were plated at a concentration of 4000/well in 96-well plates. After drug treatment, the fresh medium (100 $\mu \mathrm{L}$ per well) containing CCK-8 solution $(10 \mu \mathrm{L})$ was added to each well, followed by incubation at $37^{\circ} \mathrm{C}$ for $3 \mathrm{~h}$. Cell viability was measured at $450 \mathrm{~nm}$ using a microplate reader (Bio-Tek, USA).

\subsection{Enzyme-linked immunosorbent assay (ELISA) assay}

IL-6 and TNF- $\alpha$ levels were detected by ELISA kits (DAKEWE, China) following the protocol provided. Briefly, HGFs were plated in 6 -well plates at a concentration of $2 \times 10^{5}$ cells. These cells were supplemented with a fresh medium containing different reagents. After incubation time, HGFs culture supernatants were collected for ELISA assay. The samples were was detected at 450nm using a microplate reader (Bio-Tek, USA).

\subsection{Small interfering $R N A$ (siRNA) transfection}

$2 \times 10^{5}$ HGFs were seeded in 6-well plates before transfection. 24 hours later, cells were treated with siRNA targeting PRDX6 or a negative control siRNA using Lipo8000 (Beyotime, China) as a transfection reagent. The human PRDX6 siRNA (sequence: Sense: 5'GGAUAUCAAUGCUUACAAUTT-3';Anti-sense:5'-AUUGUAAGCAUUGAUAUCCTT$\left.3^{\prime}\right)$ was purchased from GenePharma (China). After transfection for $48 \mathrm{~h}$, the efficacy of PRDX6 silencing was analyzed by RT-qPCR and Western blotting.

\subsection{Real-time quantitative Polymerase Chain Reaction (RT-qPCR)}

Total RNA of HGFs was extracted with TRIzol (Invitrogen) and reverse-transcribed with the PrimeScript RT Reagent Kit (Takara, Japan) following the manufacturer's instructions. RT-qPCR was performed with SYBR Green Master Mix (Takara, Japan) on a Stratagene Mx3000P system (Agilent Technologies, USA). The relative gene levels were analyzed using the $2^{-\Delta \Delta C T}$ method. The sequences of examined primer are listed in Table 2.

Table 2. Primer sequences for RT-qPCR.

\begin{tabular}{ll}
\hline Gene & Primer sequence \\
\hline PRDX6 & F: 5'-ACCACTGGCAGGAACTTTGATGAG-3' \\
& R: 5'-GGCTTCTTCTTCAGGGATGGTTGG-3' \\
NRF2 & F: 5'-TCCAAGTCCAGAAGCCAAACTGAC-3' \\
& R: 5'- GGAGAGGATGCTGCTGAAGGAATC-3' \\
IL-6 & F: 5' - TTCGGTCCAGTTGCCTTCT-3' \\
\hline
\end{tabular}




\begin{tabular}{ll}
\hline \multirow{3}{*}{ TNF- $\alpha$} & R: 5'- GGTGAGTGGCTGTCTGTGTG-3' \\
& F: 5'-ATGGCGTGGAGCTGAGAGAT-3' \\
\multirow{3}{*}{-Actin } & R: 5'- TCTGGTAGGAGACGGCGATG -3' \\
& F: 5'- GCCAACACAGTGCTGTCTGG-3' \\
& R: 5'-CTCAGGAGGAGCAATGATCTTG-3' \\
\hline
\end{tabular}

\subsection{Western blotting}

Human gingival tissues and cell samples were lysed in RIPA lysis (Beyotime, China). The total protein concentration was detected by Bradford Protein Assay Kit (Beyotime, China). After that, one-fourth volume of $5 \times$ SDS loading buffer was added in protein samples and heated at $99^{\circ} \mathrm{C}$ for $10 \mathrm{~min}$. Proteins $(20 \mu \mathrm{g} / \mathrm{each})$ were separated by $10 \%$ SDSPAGE and then transferred to PVDF membranes (Millipore, USA). Membranes were blocked with 5\% BSA diluted in PBS for $1 \mathrm{~h}$ at room temperature and then incubated with primary antibodies against PRDX6, NRF2, and GAPDH overnight at $4^{\circ} \mathrm{C}$. After washing with Tris Buffered saline Tween (TBST) three times, membranes were incubated with appropriate HRP-conjugated secondary antibodies for $1 \mathrm{~h}$. After washing three times by TBST, the labeled protein was visualized by imaging system (Alliance Mini $4 \mathrm{M} ; \mathrm{UK}$ ) and quantified using ImageJ (Bethesda, USA).

\subsection{Determination of ROS generation}

Intracellular ROS in HGFs was detected using DCFH-DA (Beyotime, China). HGFs were plated in 6-well plates at a concentration of $1.5 \times 10^{5}$ cells and treated with different test compounds. Cells of each group were harvested and incubated with $500 \mu \mathrm{L}$ DCFHDA reagent $(10 \mu \mathrm{M})$ at $37^{\circ} \mathrm{C}$ for $30 \mathrm{~min}$. Then cells were washed in PBS, resuspended in $500 \mu \mathrm{L}$ PBS and analyzed using flow cytometry (CytExpert, USA). Samples can be excited at $488 \mathrm{~nm}$, and the data were detectable in the FL1 channel.

\subsection{Statistical analysis}

Representative data were collected from three or more times independent experiments. All statistics were analyzed using Graphpad Prism 9. Data were displayed as mean \pm standard deviation (SD). Statistical significance between the groups was described as follows: ${ }^{*} \mathrm{p}<0.05,{ }^{* *} \mathrm{p}<0.01,{ }^{* * *} \mathrm{p}<0.001$.

\section{Results}

3.1. The expression of PRDX6 and NRF2 decreased in inflamed gingival tissues

We first determined the effect of periodontitis on the expression levels of PRDX6 and NRF2. H\&E staining results showed that the gingival tissues of periodontitis patients exhibited inflammatory cell infiltration, while healthy gingival tissues exhibited healthy architecture (Fig. 1a). The IHC and Western blotting results showed that PRDX6 decreased in periodontitis gingival tissues (Fig. 1b, d). NRF2 is a primary transcription factor that can activate antioxidant genes to defend against oxidative stress. We also assessed the NRF2 expression level in periodontitis and normal gingival tissue. As shown in Fig. 1c and 1d, NRF2 was decreased significantly in inflamed gingival tissues than in control. Therefore, the oxidative capacity was reduced and led to tissue damage in periodontitis. 
a
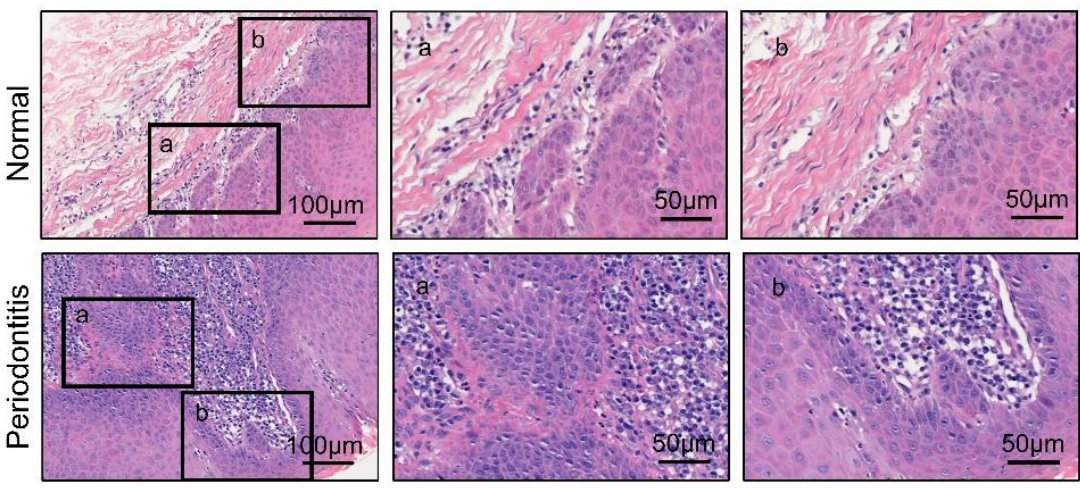

H\&E

b
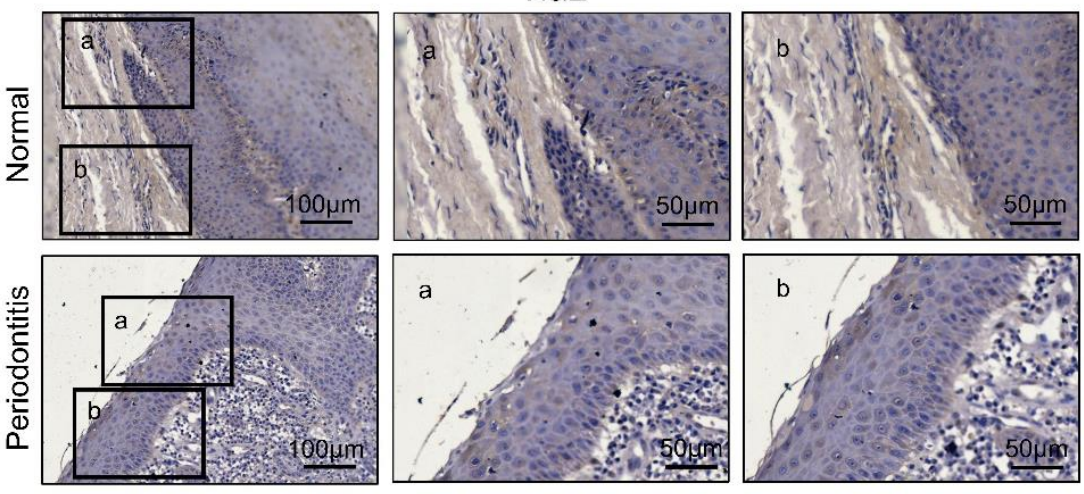

PRDX6

C
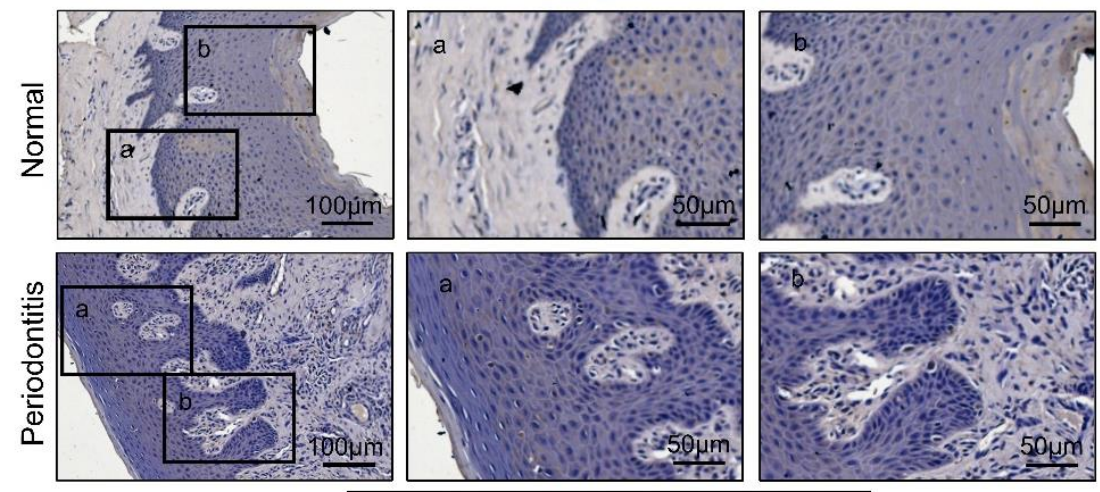

NRF2

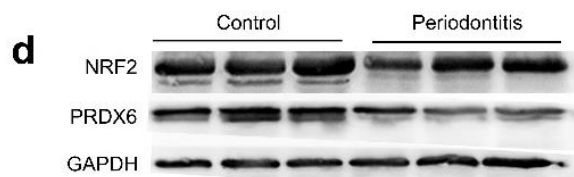

Figure 1. The expression of PRDX6 and NRF2 decreased in inflamed gingival tissues. (a) H\&E stain in human periodontitis gingival tissues and normal tissues. (b-d) The expression of PRDX6 and NRF2 in control and inflammatory gingival tissues was assessed by IHC and Western blotting.

\subsection{The expression of PRDX6 and NRF2 in LPS-induced HGFs}

Then we investigated the effect of LPS on the expression levels of PRDX6 and NRF2 in HGFs. We first analyzed the consequence of LPS treatment in different concentrations on inflammatory cytokines in HGFs. As shown in Fig. 2a, RT-qPCR results confirmed that the levels of IL- 6 and TNF- $\alpha$ were upregulated in a dose-dependent manner and climbed to the top with $10 \mu \mathrm{g} / \mathrm{mL}$ LPS treatment. Then we evaluated the influence of LPS treatment $(10 \mu \mathrm{g} / \mathrm{mL})$ for different hours on inflammatory cytokines in HGFs. As shown in Fig. 2a, IL-6 and TNF- $\alpha$ were elevated significantly after $10 \mu \mathrm{g} / \mathrm{mL}$ LPS treatment for $12 \mathrm{~h}$. We also explored the effect of LPS treatment on the expression of PRDX6 and NRF2 in HGFs. The Western blotting and RT-qPCR results confirmed that the expression levels of PRDX6 
and NRF2 were also increased in a dose-dependent manner under LPS treatment in different concentrations. The up-regulation of PRDX6 and NRF2 was observed from 12 to 24 $h$, and the down-regulation was observed at $72 \mathrm{~h}$ (Fig. 2e and 2f). In brief, the expression of PRDX6 and NRF2 was first increased and eventually decreased under the long-term LPS treatment. Of note, PRDX6 and inflammatory cytokines both significantly increased when HGFs were treated with $10 \mu \mathrm{g} / \mathrm{mL}$ LPS for 12 hours. This treatment was used to guide the subsequent study.

a

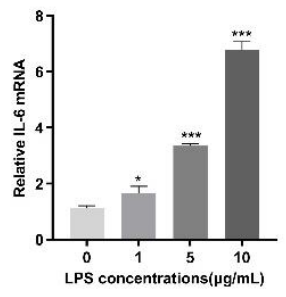

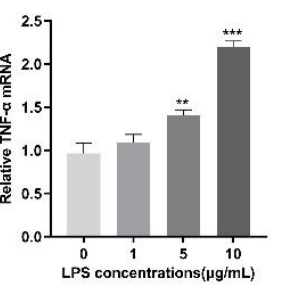
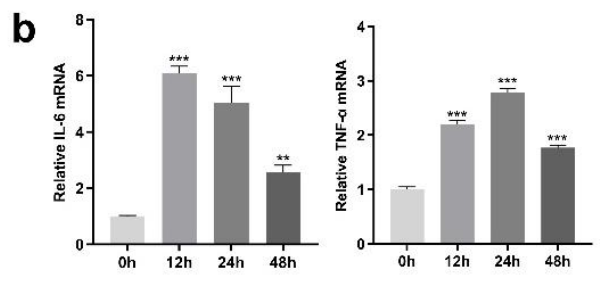

C
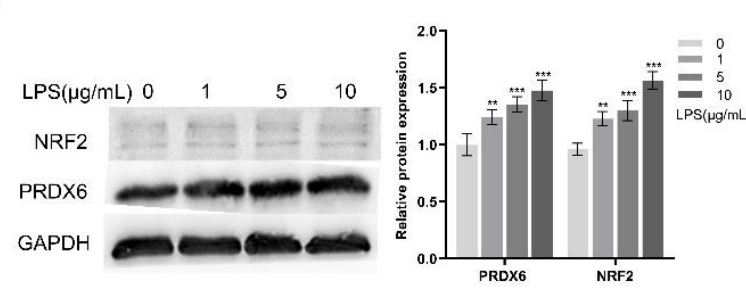

e
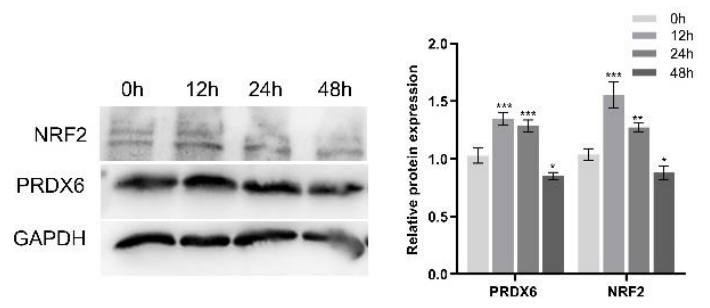

d

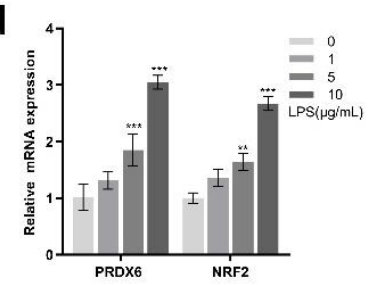

f

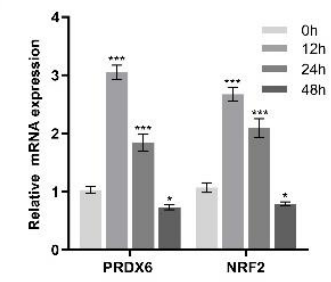

Figure 2. The expression of PRDX6 and NRF2 in LPS-induced HGFs. (a, b) HGFs were treated with LPS $(0,1,5,10 \mu \mathrm{g} / \mathrm{mL})$ for $12 \mathrm{~h}$ or treated with $10 \mu \mathrm{g} / \mathrm{mL}$ LPS for different time $(0,12,24$, and $48 \mathrm{~h})$, then the mRNA expression of IL- 6 and TNF- $\alpha$ were assessed by RT-qPCR. (c, d) HGFs were treated with LPS $(0,1,5,10 \mu \mathrm{g} / \mathrm{mL})$ for $12 \mathrm{~h}$, the protein and mRNA expression of PRDX6 and NRF2 were assessed by Western blotting and RT-qPCR. (e, f) HGFs were treated with $10 \mu \mathrm{g} / \mathrm{mL}$ LPS for different time $(0,12,24$, and $48 \mathrm{~h})$, the protein and mRNA expression of PRDX6 and NRF2 were assessed by Western blotting and RT-qPCR. Data were displayed as mean \pm SD. ${ }^{*} \mathrm{p}<0.05 ;{ }^{* *} \mathrm{p}<0.01 ;{ }^{* *} \mathrm{p}<$ 0.001 .

\subsection{PRDX6 protected HGFs from LPS-induced inflammatory cytokines and ROS}

To explore the role of PRDX6 in periodontitis, we investigated the levels of inflammatory cytokines and ROS in LPS induced PRDX6 knockdown HGFs. After the transfection of PRDX6 siRNA, PRDX6 expression was significantly decreased (Fig. 3a, b). Subsequently, the cell viability was quantified in PRDX6 knockdown cells with treatment of LPS. The cell viability was reduced in PRDX6 knockdown HGFs compared to control knockdown after LPS treatment. The results of RT-qPCR and ELISA showed that PRDX6 knockdown could not increase the levels of IL-6 and TNF- $\alpha$ than control knockdown. However, it significantly enhanced the expression of inflammatory cytokines in LPS induced HGFs (Fig. 3c, d). Furthermore, PRDX6 knockdown could not upregulate the ROS, while it increased LPS induced ROS in HGFs. Thus, the knockdown of PRDX6 amplifies the LPS induced inflammation and oxidative stress. 
$\mathbf{a}$
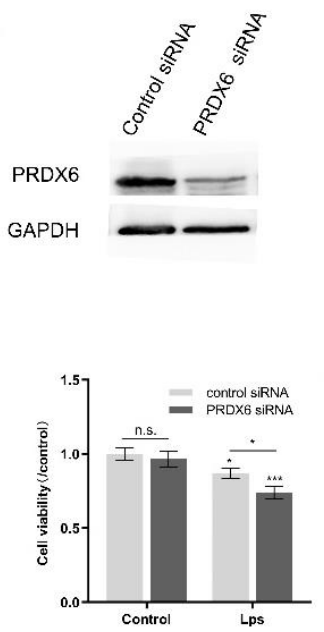

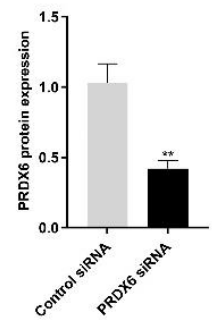

d

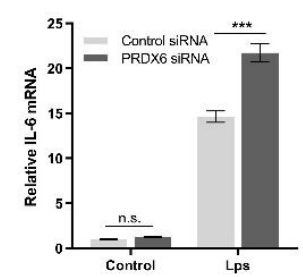

b
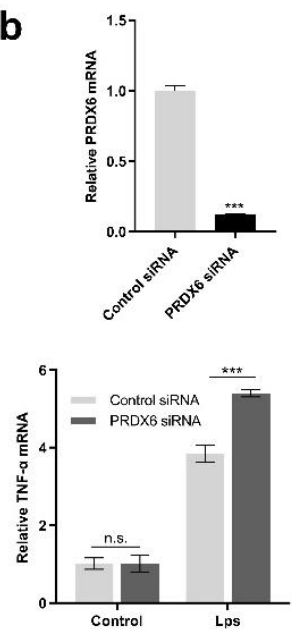

Multi-sample : P1

e
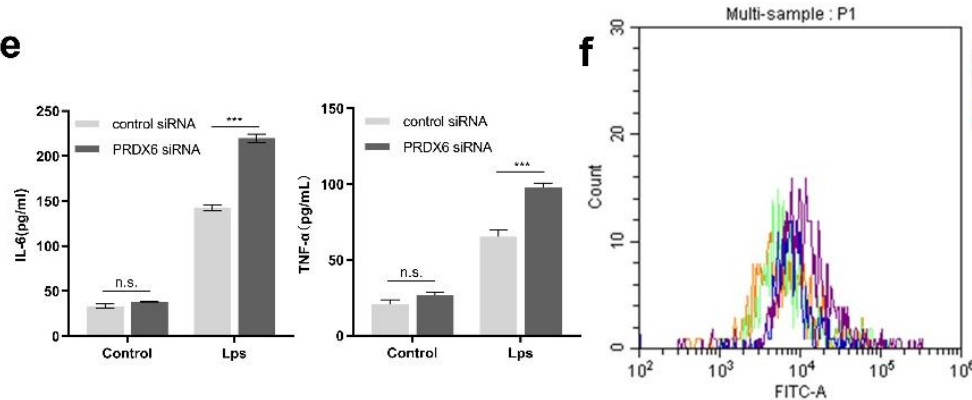

Figure 3. PRDX6 protected HGFs from LPS-induced inflammatory cytokines and ROS. (a, b) The mRNA and protein expression levels of PRDX6 were assessed after infection of PRDX6 siRNA for $48 \mathrm{~h}$ by RT-qPCR and Western blotting. (c) HGFs were stimulated by LPS $(10 \mu \mathrm{g} / \mathrm{mL})$ for $12 \mathrm{~h}$ after transfection for $48 \mathrm{~h}$, and the cell viability was assayed by CCK8 assay. (d, e) The expression levels of IL- 6 and TNF- $\alpha$ were detected by RT-qPCR and ELISA. (f) ROS production of each group was assayed by flow cytometry. Data were displayed as mean \pm SD. ${ }^{*} p<0.05 ;{ }^{* *} p<0.01$; ${ }^{* * *} p<0.001 ; n . s$.: no statistic difference.

\subsection{The inhibition of PRDX6-PLA2 alleviated LPS-induced inflammatory cytokines and ROS}

Because the specific inhibitors of the PLA2 activity of PRDX6 (MJ33) are commercially available, we mainly focused on whether the PLA2 activity of the multi-tasking enzyme PRDX6 could influence inflammation and oxidative stress. Fig. 4a showed that the cell viability was upregulated in the MJ33+LPS group compared with the LPS group. As shown in Fig. 4b and 4c, MJ33 treatment could not affect the levels of IL- 6 and TNF- $\alpha$ in HGFs. However, the LPS-induced inflammatory cytokines were down-regulated by MJ33 co-treatment. In addition, the inhibition of PLA2 activity decreased the production of LPSinduced ROS (Fig. 4d). Therefore, PRDX6 could partially promote inflammation and oxidative stress via PLA2 activity. 
a

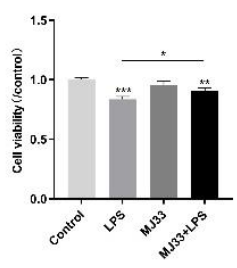

C

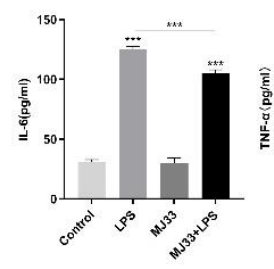

b
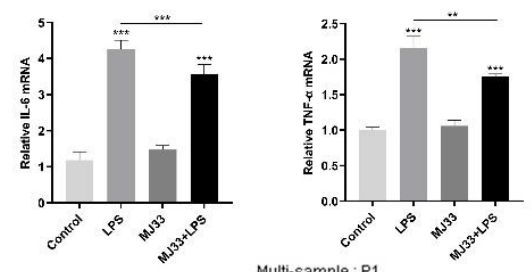

d

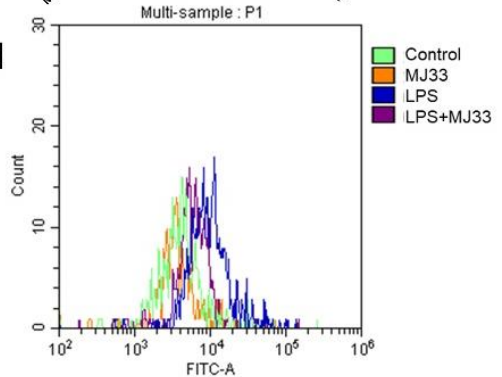

Figure 4. The inhibition of PRDX6-PLA2 alleviated LPS-induced inflammatory cytokines and ROS. (a) HGFs were stimulated by LPS $(10 \mu \mathrm{g} / \mathrm{mL})$ with or without MJ33 $(5 \mu \mathrm{M})$ for $12 \mathrm{~h}$, the cell viability was assayed by CCK8 assay. (b, c) The expression of IL- 6 and TNF- $\alpha$ of each group was detected by RT-qPCR and ELISA. (d) ROS production of each group was assayed by flow cytometry. Data were displayed as mean \pm SD. ${ }^{*} \mathrm{p}<0.05 ;{ }^{* *} \mathrm{p}<0.01 ;{ }^{* * *} \mathrm{p}<0.001$; n.s.: no statistic difference.

\subsection{PRDX6 regulated by NRF2 ameliorated LPS-induced inflammatory cytokines and ROS}

PRDX6 served as a downstream target gene of NRF2. To further confirm the effect of NRF2 regulation on the expression of PRDX6, we used NRF2 inhibitor ML385 to inhibit the NRF2. The cell viability under the co-treatment of ML385 and LPS could be further decreased than cells only treated with LPS (Fig. 5a). The RT-qPCR results confirmed that the expression levels of NRF2 and PRDX6 were decreased with the ML385 treatment than control cells. The co-treatment of LPS and ML385 reduced the expression of NRF2 and PRDX6 compared with the LPS group (Fig. 5b). As shown in Fig. 5c and 5d, ML385 treatment could not initialize the expression levels of IL- 6 and TNF- $\alpha$, while it could enhance LPS-induced inflammatory cytokines in HGFs. Furthermore, LPS-induced ROS were improved in the meantime by ML385 co-treatment (Fig. 5e). Therefore, these results indicated that NRF2 could regulate the expression of PRDX6 to protect HGFs against inflammation and oxidative stress.

a

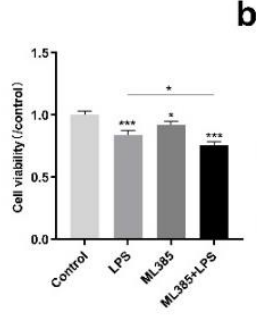

d

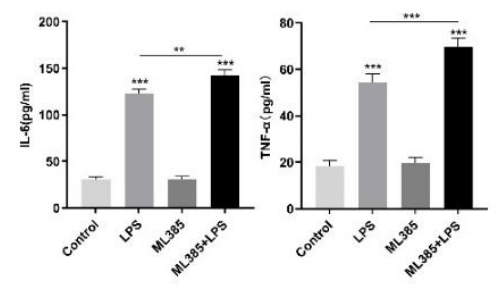

b

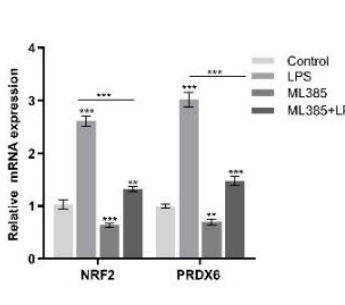

e

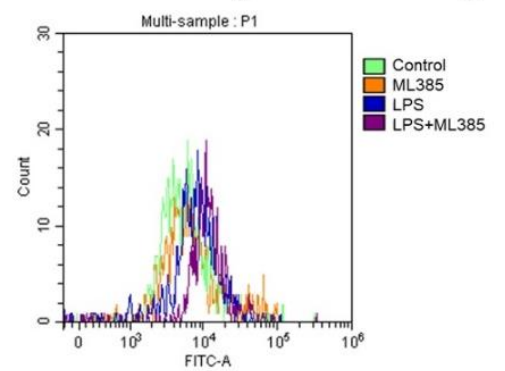

c

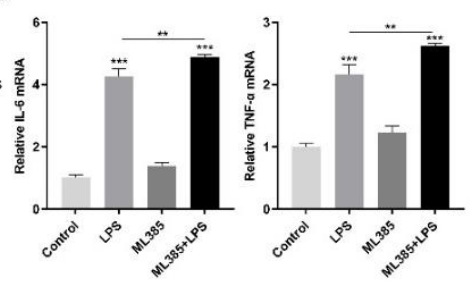

Figure 5. PRDX6 regulated by NRF2 ameliorates LPS-induced inflammatory cytokines and ROS. (a) HGFs were stimulated by LPS $(10 \mu \mathrm{g} / \mathrm{mL})$ with or without ML385 $(5 \mu \mathrm{M})$ for $12 \mathrm{~h}$, and the cell viability was assayed by CCK8 assay. (b) The mRNA expression of NRF2 and PRDX6 of each group was assessed by RT-qPCR. (c, d) The expression levels of IL- 6 and TNF- $\alpha$ of each group were 
detected by RT-qPCR and ELISA. (e) ROS production of each group was assayed by flow cytometry. Data were displayed as mean \pm SD. ${ }^{*} \mathrm{p}<0.05 ;{ }^{* *} \mathrm{p}<0.01 ;{ }^{* * *} \mathrm{p}<0.001$

\section{Discussion}

Even though the LPS-induced periodontitis model has been established for years and is widely used in pre-clinical studies, the precise mechanisms remain unclear. It is reported that HGFs can be activated by LPS and release significant inflammatory factors, which contribute to periodontal tissue destruction $[25,26]$. What's more, there are still exit oxidative stress injuries because of the accumulation of oxidation products[27]. It has been reported that PRDX6 is multifunctional to scavenge peroxides and generate ROS via PLA2 activity[28]. Therefore, PRDX6 is a regulator of the cellular redox of biological systems. In this study, we mainly discussed the role of PRDX6 in LPS-induce inflammation and oxidative stress in HGFs.

PRDX6 holds an essential position in inflammatory diseases and hasn't been reported in periodontitis. We found that the expression level of PRDX6 was decreased in inflamed gingival tissues due to severe oxidative damage in chronic periodontitis. HGFs are the most abundant cells in periodontal tissue and the critical barrier against bacterial infection. In HGFs, the expression of PRDX6 was increased considerably to defend against the LPS-induced oxidative damage at first, while it eventually decreased and lost the protective effect.

Interestingly, PRDX6 has been recently identified as the regulator of redox balance. We then identified the inflammatory cytokines and ROS in PRDX6-silenced cells. PRDX6 knockdown elevated the levels of inflammatory cytokines released by HGFs, which indicated that PRDX6 plays a protective role in LPS-induced inflammation. Furthermore, more ROS production could be observed in silencing HGFs treated with LPS and participates in the onset and amplification of inflammation. PRDX6 could play peroxidase activity to act as an antioxidant reducing short-chain hydroperoxide. Thus, PRDX6 limits oxidative stress in LPS-induced HGFs. It has been reported that PRDX6 plays a paradoxical role in inflammatory and immune responses. For example, in the LPS-induced acute kidney injury model, PRDX6-overexpressed mice showed less renal apoptosis and leukocyte infiltration than wild mice [29]. Conversely, PRDX6 lost its antioxidant activity in aortic lesions or aneurysms, characterized by high inflammation and oxidative stress [30]. Our results showed that PRDX6 could alleviate ROS and has a protective effect on periodontitis. However, we only demonstrated this mechanism in vitro experiments, and the vivo model was necessary to analyze the essential role of PRDX6 in the future.

Notably, PRDX6 is a peroxiredoxin that owns multiple enzyme activities, peroxidase, PLA2, and LPCAT. It has been reported that blocking the PLA2 activity of PRDX6 by MJ33 enhanced ROS [31]. In our study, the inhibition of PRDX6-PLA2 activity down-regulated the production of LPS-induced ROS and inflammatory cytokines. The potential mechanism for MJ33 to alleviate the inflammation and oxidative stress through the inhibition of the PLA2 activity to block NOX2 activation. NOX2 is the enzyme responsible for ROS generation associated with inflammation [32]. It has been reported that enhanced NOX2 activity is most likely one of the ROS sources in periodontal tissues [33]. PRDX6-PLA2 could play a protective role in reducing phospholipid hydroperoxides and repairing peroxidized cell membranes. However, it is less than the protective role of PHGPx activity and LPCAT activity. This result was in line with the study that blocking PRDX6-PLA2 activity could attenuate lungs damage, presumably by inhibiting oxidative stress and preventing the amplification of lungs inflammation [32]. Therefore, inhibiting PRDX6-PLA2 activity alleviated the LPS-induced inflammation and oxidative injury in HGFs.

Furthermore, the NRF2 is a vital regulator of the cellular antioxidant response, as many of its downstream genes prevent oxidative stress [34]. The activated NRF2 can be transported to the nucleus and subsequently bind to ARE to improve the transcription of some antioxidant genes, such as HO-1 and NQO1[35, 36]. In our study, the 
downregulation of NRF2 was observed in inflammatory gingival tissues compared to the normal gingival tissues. What's more, NRF2 was intensely activated to promote HGFs at first and eventually was decreased with long-term LPS treatment, which is in line with the result of PRDX6. With the treatment of NRF2 inhibitor ML385, the expression of PRDX6 was down-regulated. In addition, the levels of inflammatory cytokines and ROS were increased as ML385 eliminated the protective role of NRF2. Therefore, the NRF2 could play a protective role in LPS induced-HGFs. However, this study only explored the primary mechanism between PRDX6 and NRF2 (Fig. 6), and the deep mechanism still needs to be further explored.

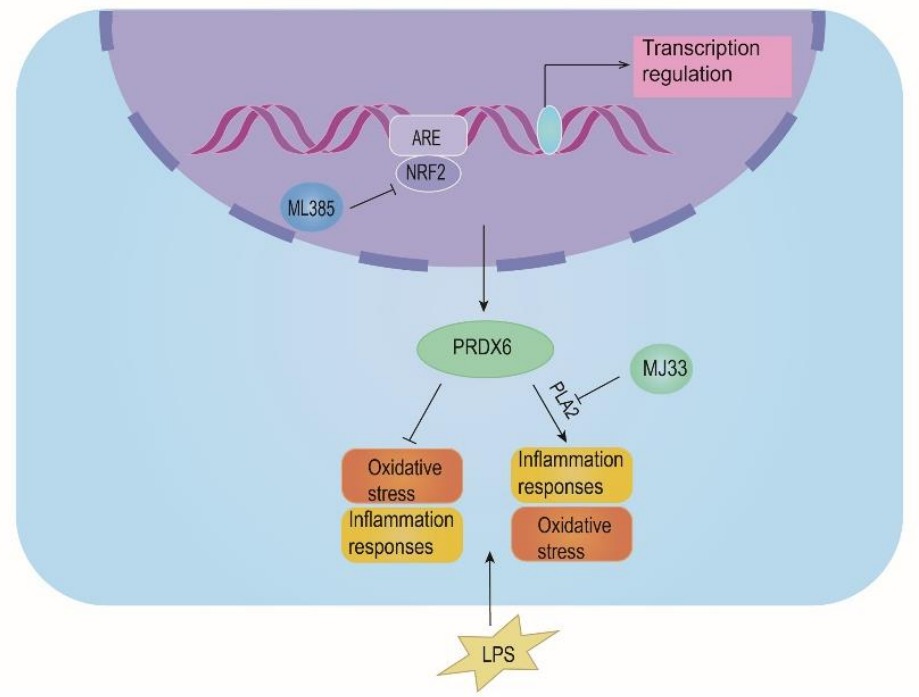

Figure 6. The role of PRDX6 in LPS-induce HGFs. PRDX6, regulated by the NRF2 signaling, alleviates LPS- induced inflammatory response and oxidative stress in human gingival fibroblasts.

\section{Conclusions}

Collectively, we mainly analyzed the role of PRDX6 in LPS-induced inflammatory response and oxidative stress. The present study demonstrated that PRDX6 could alleviate the LPS-induced inflammation and oxidative stress in HGFs. The NRF2 signaling is an essential antioxidant response pathway that regulates the expression of PRDX6, which also affects the inflammation reaction in periodontitis. Therefore, this study provided new insight into periodontitis therapy targeting PRDX6 and oxidative stress.

Author Contributions: Conceptualization, Methodology, and Writing-Original draft preparation: Wen-ying Yang; Formal analysis: Xiang Meng; Investigation: Yue-rong Wang; Writing-Reviewing and Editing: Qing-qing Wang; Validation: Xin He; Visualization: Xiao-yu Sun; Resources: Nan Cheng; Supervision: Lei Zhang.

Funding: This research was funded by the Natural Science Foundation of Anhui Province (grant number 1908085QH328) and the University Natural Science Research Project of Anhui Province ((grant number KJ2018A0203).

Institutional Review Board Statement: The study was conducted according to the guidelines of the Declaration of Helsinki, and approved by the Health Human Research Ethics Committee of the Stomatology Hospital affiliated to Anhui Medical University (Permit No. 20200836).

Informed Consent Statement: Written informed consent has been obtained from the patients.

Conflicts of Interest: The authors declare no conflict of interest. 
1. Bawaskar, H.S. and P.H. Bawaskar, Oral diseases: a global public health challenge. Lancet, 2020. 395(10219): p. 185-186.

2. Kinane, D.F., Causation and pathogenesis of periodontal disease. Periodontol 2000, 2001. 25: p. 8-20.

3. Kassebaum, N.J., et al., Global burden of severe periodontitis in 1990-2010: a systematic review and meta-regression. J Dent Res, 2014. 93(11): p. 1045-53.

4. Lei, L., et al., Porphyromonas gingivalis lipopolysaccharide alters atherosclerotic-related gene expression in oxidized low-densitylipoprotein-induced macrophages and foam cells. J Periodontal Res, 2011. 46(4): p. 427-37.

5. $\quad$ Bartold, P.M. and T.E. Van Dyke, Periodontitis: a host-mediated disruption of microbial homeostasis. Unlearning learned concepts. Periodontol 2000, 2013. 62(1): p. 203-17.

6. Brunner, J., et al., The capsule of Porphyromonas gingivalis reduces the immune response of human gingival fibroblasts. BMC Microbiol, 2010. 10: p. 5.

7. Hajishengallis, G., Periodontitis: from microbial immune subversion to systemic inflammation. Nat Rev Immunol, 2015. 15(1): p. 30-44.

8. Wang, G.P., Defining functional signatures of dysbiosis in periodontitis progression. Genome Med, 2015. 7(1): p. 40.

9. Buczko, P., A. Zalewska, and I. Szarmach, Saliva and oxidative stress in oral cavity and in some systemic disorders. J Physiol Pharmacol, 2015. 66(1): p. 3-9.

10. Hernández-Ríos, P., et al., Oxidative Stress in the Local and Systemic Events of Apical Periodontitis. Front Physiol, 2017.8 : p. 869. 11. Kim, D.Y., et al., N-acetylcysteine prevents LPS-induced pro-inflammatory cytokines and MMP2 production in gingival fibroblasts. Arch Pharm Res, 2007. 30(10): p. 1283-92.

12. Zhao, Y., et al., Periodontitis-level butyrate-induced ferroptosis in periodontal ligament fibroblasts by activation of ferritinophagy. Cell Death Discov, 2020. 6(1): p. 119.

13. Chen, H., et al., Recombinant Klotho Protects Human Periodontal Ligament Stem Cells by Regulating Mitochondrial Function and the Antioxidant System during H(2)O(2)-Induced Oxidative Stress. Oxid Med Cell Longev, 2019. 2019: p. 9261565.

14. Huang, C., et al., Eldecalcitol Inhibits LPS-Induced NLRP3 Inflammasome-Dependent Pyroptosis in Human Gingival Fibroblasts by Activating the Nrf2/HO-1 Signaling Pathway. Drug Des Devel Ther, 2020. 14: p. 4901-4913.

15. Hagiu, A., et al., Dose-dependent green tea effect on decrease of inflammation in human oral gingival epithelial keratinocytes: in vitro study. Clin Oral Investig, 2020. 24(7): p. 2375-2383.

16. Yang, D., et al., Deletion of peroxiredoxin 6 potentiates lipopolysaccharide-induced acute lung injury in mice. Crit Care Med, 2011. 39(4): p. 756-64.

17. Yun, H.M., et al., PRDX6 promotes lung tumor progression via its GPX and iPLA2 activities. Free Radic Biol Med, 2014. 69: $\mathrm{p}$. $367-76$.

18. Wahlig, S., M. Lovatt, and J.S. Mehta, Functional role of peroxiredoxin 6 in the eye. Free Radic Biol Med, 2018. 126: p. 210-220.

19. Hattori, F. and S. Oikawa, Peroxiredoxins in the central nervous system. Subcell Biochem, 2007. 44: p. 357-74.

20. Pacifici, F., et al., Peroxiredoxin 6, a novel player in the pathogenesis of diabetes. Diabetes, 2014. 63(10): p. 3210-20.

21. Kang, S.W., I.C. Baines, and S.G. Rhee, Characterization of a mammalian peroxiredoxin that contains one conserved cysteine. J Biol Chem, 1998. 273(11): p. 6303-11.

Pedrajas, J.R., et al., Glutathione Is the Resolving Thiol for Thioredoxin Peroxidase Activity of 1-Cys Peroxiredoxin Without Being Consumed During the Catalytic Cycle. Antioxid Redox Signal, 2016. 24(3): p. 115-28.

Aboodi, G.M., et al., Salivary Cytoprotective Proteins in Inflammation and Resolution during Experimental Gingivitis--A Pilot Study. Front Cell Infect Microbiol, 2015. 5: p. 92.

24. Zou, Y., et al., IncRNA expression signatures in periodontitis revealed by microarray: the potential role of lncRNAs in periodontitis pathogenesis. J Cell Biochem, 2015. 116(4): p. 640-7.

25. Cochran, D.L., Inflammation and bone loss in periodontal disease. J Periodontol, 2008. 79(8 Suppl): p. 1569-76. 
26. Kardachi, B., G.N. Wolffe, and J.D. Manson, Gingival inflammation and bone loss in periodontal disease. J Clin Periodontol, 1979. 6(4): p. 252-9.

27. Liu, C., et al., The Role of Reactive Oxygen Species and Autophagy in Periodontitis and Their Potential Linkage. Front Physiol, 2017. 8: p. 439.

28. Manevich, Y. and A.B. Fisher, Peroxiredoxin 6, a 1-Cys peroxiredoxin, functions in antioxidant defense and lung phospholipid metabolism. Free Radic Biol Med, 2005. 38(11): p. 1422-32.

29. Lee, D.H., et al., Peroxiredoxin 6 overexpression attenuates lipopolysaccharide-induced acute kidney injury. Oncotarget, 2017. 8(31): p. 51096-51107.

30. Chhunchha, B., et al., Aberrant sumoylation signaling evoked by reactive oxygen species impairs protective function of Prdx6 by destabilization and repression of its transcription. Febs j, 2014. 281(15): p. 3357-81.

31. Chatterjee, S., et al., Peroxiredoxin 6 phosphorylation and subsequent phospholipase A2 activity are required for agonist-mediated activation of NADPH oxidase in mouse pulmonary microvascular endothelium and alveolar macrophages. J Biol Chem, 2011. 286(13): p. 11696-706.

32. Benipal, B., et al., Inhibition of the phospholipase A2 activity of peroxiredoxin 6 prevents lung damage with exposure to hyperoxia. Redox Biol, 2015. 4: p. 321-7.

33. Giannopoulou, C., K.H. Krause, and F. Müller, The NADPH oxidase NOX2 plays a role in periodontal pathologies. Semin Immunopathol, 2008. 30(3): p. 273-8.

34. Chiu, A.V., et al., The Role of NrF2 in the Regulation of Periodontal Health and Disease. J Dent Res, 2017. 96(9): p. 975-983.

35. Chhunchha, B., E. Kubo, and D.P. Singh, Clock Protein Bmal1 and Nrf2 Cooperatively Control Aging or Oxidative Response and Redox Homeostasis by Regulating Rhythmic Expression of Prdx6. Cells, 2020. 9(8).

36. Raghunath, A., et al., Antioxidant response elements: Discovery, classes, regulation and potential applications. Redox Biol, 2018. 17: p. 297-314. 